\title{
Integración de Sistemas Solares Fotovoltaicos en el Sector Camaronero Intensivo y Extensivo del Ecuador: Caso de Estudio en la Provincia de El Oro
}

\author{
Pesantez, Juan-Pablo $^{1}$ (D) ; Ríos-Villacorta, Alberto $^{2}$ iD ; González-Redrován, Javier $^{1}$ iD \\ ${ }^{1}$ Universidad Católica de Cuenca, Facultad de Ingeniería de Sistemas, Eléctrica y Electrónica, Cuenca - Ecuador \\ ${ }^{2}$ Universidad Técnica de Ambato, Facultad de Ingeniería en Sistemas, Electrónica e Industrial, Ambato - Ecuador
}

\begin{abstract}
Resumen: Actualmente, la integración de sistemas solares fotovoltaicos en las redes de distribución para el suministro eléctrico directo a usuarios industriales permite reducir significativamente los costos de operación e incrementar la competitividad de los sectores productivos. En este sentido, los sistemas solares fotovoltaicos podrían suministrar un importante porcentaje de las necesidades eléctricas de las empresas camaroneras de forma económica y fiable. Las fincas camaroneras se localizan generalmente en sitios remotos, cerca de las costas e incluso en islas, sin conexión al sistema eléctrico nacional. La construcción de redes eléctricas de suministro eléctrico resultaría excesivamente costosa. Así, los motores diésel de combustión interna se encargan de garantizar el suministro de la demanda energética en esta industria. Los resultados del presente estudio demuestran que la integración de sistemas fotovoltaicos de generación solar puede satisfacer parcialmente la demanda eléctrica de los cultivos intensivos y extensivos de camarones de la provincia de El Oro. El estudio se lo realiza en Puerto Pitahaya perteneciente al cantón Arenillas, donde se comprueba la existencia de un excepcional recurso solar en promedio $133.02 \mathrm{kWh} / \mathrm{m}^{2}$ - mes, el costo de inversión por cada $\mathrm{kW}$ de esta tecnología es de $\$ 415,8$ lo cual representa un precio competitivo, así mismo la madurez de la tecnología solar garantizan una rápida recuperación de la inversión, un suministro eléctrico de elevada fiabilidad y una sustancial mejora de la eficiencia energética de la producción de las empresas del sector camaronero del Ecuador.
\end{abstract}

Palabras clave: Energía, panel, fotovoltaico, eficiencia, acuacultura.

\section{Integration of Photovoltaic Solar Systems in the Intensive and Extensive Shrimp Sector of Ecuador: El Oro Province Study Case}

\begin{abstract}
At present, the integration of solar photovoltaic systems into electricity distribution networks that supply energy to industrial users, allows to significantly reduce operating costs and increase competitiveness in productive sectors. In this context, solar photovoltaic systems could satisfy a significant percentage of the electricity needs of shrimp companies in a cost-effective and reliable way. Shrimp farms are generally located in remote places, near the coast and even on islands, without access to the national electrical grid. The construction of electricity supply networks would be excessively expensive. Thus, diesel internal combustion engines are responsible for meeting shrimp companies energy demands. The results of this study demonstrate that the integration of solar photovoltaic systems can partially satisfy the energy demands of intensive and extensive shrimp farming in El Oro province. The study is carried out in Puerto Pitahaya belonging to the Arenillas city, where the existence of an exceptional solar resource is verified on average $133.02 \mathrm{kWh} / \mathrm{m}^{2}--\mathrm{month}$, the investment cost for each $\mathrm{kW}$ of this technology is $\$ 415.8$, which represents a competitive price, as well as the maturity of solar technology guarantee a quick return on investment, a highly reliable electricity supply and a substantial improvement in the energy efficiency of the production of companies in the shrimp sector of Ecuador.
\end{abstract}

Keywords: Photovoltaic, Solar, Energy, energy efficiency, aquaculture.

\section{INTRODUCCIÓN}

En la década de los años 60 se inició la producción camaronera del Ecuador, en el cantón Santa Rosa, provincia de El Oro. La producción de camarón en cautiverio, en piscinas junto a esteros, fue un emprendimiento de los agricultores de la zona. En la provincia de El Oro, en el año 1974, se dedicaban al cultivo de este crustáceo alrededor de 600 hectáreas. La actividad de producción de camarón se extendió a la provincia del Guayas, en la que se convirtió en un negocio muy rentable gracias a la abundancia de esteros y salitrales (Intriago, 2016).

En la actualidad, el sector camaronero representa un rubro muy importante para el crecimiento económico del Ecuador. En el 
tercer trimestre del año 2018, la economía del país experimentó un crecimiento del 1,4\% en relación al segundo trimestre del mismo año según cifras del Banco Central del Ecuador, BCE. En tanto que, sólo en el sector acuícola, se constató un crecimiento interanual de 7,4\%. El importante crecimiento del sector camaronero se sustentó por las innovaciones tecnológicas introducidas en la producción de tipo intensivo y extensivo. En el año 2018, las exportaciones del sector camaronero alcanzaron un volumen superior a 130 mil toneladas, que representó un incremento interanual del 18\% (Haz, 2019a).

La rentabilidad del sector acuícola camaronero depende directamente del nivel de tecnificación de los procesos de producción y de la dependencia de los precios de combustibles fósiles, además del constante peligro de contaminación por el uso de derivados de petróleo. El principal negocio del sector camaronero es la producción de camarón, sin embargo, es necesario considerar la energía como un recurso gestionable de forma inteligente mediante la introducción de acciones de eficiencia energética, con la aplicación de la norma ISO 50001. Una administración responsable de los recursos energéticos implica una significativa mejora de la productividad. Asimismo, es importante que las prácticas de ahorro y eficiencia energética se acompañen con la expansión de la infraestructura en las industrias, configurando una oportunidad potencial para el crecimiento de las industrias (McKane, 2009).

El Plan Nacional de Eficiencia Energética del Ecuador en el horizonte 2016 - 2035, PLANEE, fomenta la instalación de sistemas renovables de generación eléctrica, la integración de programas de eficiencia energética, así como la implementación de la certificación de la norma de eficiencia energética, ISO 50001; sin embargo, no considera impulsar el sector acuícola (Gaudry, Godoy-Vaca, Espinoza, Fernández, \& Lobato-Cordero, 2019). Adicionalmente, la inminente eliminación del subsidio a los combustibles fósiles para el sector camaronero, establecido por el gobierno del Ecuador en enero de 2019, que garantiza un precio diferenciado para pequeños medianos y grandes productores, desencadenará un aumento significativo en los costos de producción del sector. En este sentido, es de especial interés para el sector camaronero el desarrollo de propuestas de integración de nuevas fuentes renovables de energía y de optimización de recursos energéticos en los procesos de producción, que permitan desacoplar el suministro energético de los combustibles fósiles (Haz, 2019b).

\section{SUSTENTABILIDAD DEL SECTOR ACUÍCOLA}

En el contexto económico la acuacultura es una actividad que genera grandes ganancias a nivel mundial, los retornos de las inversiones son relativamente rápidos lo cual hace atractivo el negocio; sin embargo, casi nunca se toma en cuenta las pérdidas ecológicas y económicas resultantes de la degradación del ambiente, en este sentido el gobierno debe jugar un papel fundamental para regular las prácticas de esta actividad de tal forma que se pueda garantizar buenas condiciones de vida para las generaciones futuras (Assad \& Bursztyn, 2000).
En las últimas décadas en Ecuador se ha experimentado un rápido crecimiento del sector acuícola, que no ha sido debidamente reglamentado, regulado y controlado, dando como resultado la aparición de cultivos de camarones de tipo intensivo sin planificación técnica y ambiental adecuada provocando un significativo número de proyectos que pierden rentabilidad e incrementan alarmante las cifras de desocupación en las provincias camaroneras (Assad \& Bursztyn, 2000); (Vieira ${ }^{1}$, de Oliveira, Crispim, \& Cunha, 2016).

La actividad económica de producción de camarones en el contexto social es muy importante, ya que representa un segmento grande de la ocupación nacional. La generación de puestos de trabajo y el gran volumen de exportación de alimentos altos en proteína generan un impacto socioeconómico muy positivo (Assad \& Bursztyn, 2000). En el contexto político, el Estado ecuatoriano debería ser capaz de promover el desarrollo sostenible del sector camaronero con la elaboración e implementación de regulaciones gubernamentales y políticas dirigidas al establecimiento de normas e instrumentos técnicos de ahorro y eficiencia energética (Assad \& Bursztyn, 2000).

\subsection{Producción de camarón}

La producción de camarones se inicia con la preparación del terreno y realización de obras civiles para la construcción de piscinas y estabilización de muros. Se requiere un suelo limoarcilloso de mínima filtración o cubierto por membrana. La preparación de las piscinas para la siembra incluye, además, el uso de fertilizantes y desinfectantes. La larva de camarón recibe un tratamiento de maduración por medio de un proceso de raceways o precriaderos, que consiste en la adaptación en estanques de menor tamaño con control de parámetros como $\mathrm{PH}$, salinidad, temperatura, oxigeno, bacterias, etc. Posteriormente, se realiza el cultivo de la post-larva para la cría y engorde en las piscinas, durante 90 y 120 días, según el tamaño de camarón que se desee. El cultivo de la post-larva exige un recambio de agua y el empleo de sistemas de aireación de 24 horas, en función de la densidad de la siembra, que garantice niveles adecuados de oxígeno para la supervivencia del camarón. Finalmente, se realiza la cosecha de forma manual o con cosechadoras, que deberá, recoger los camarones sin causarles daño ya sea por manipulación o estrés, véase Figura 1 (Aguilar \& Parrales, 2015); (Ochoa, 2016).
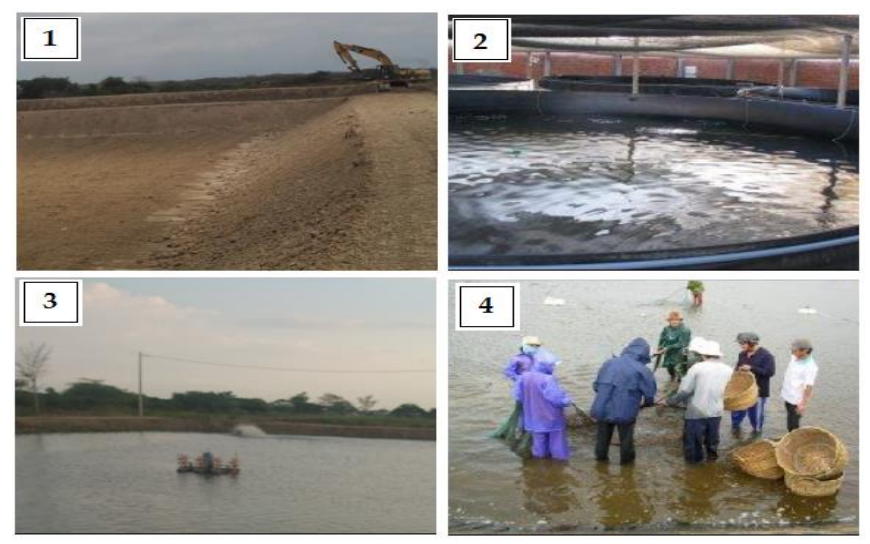

Figura 1. Fases de la producción de camarón en el Ecuador 


\section{ENERGÍAS RENOVABLES APLICADAS AL SECTOR ACUÍCOLA}

Las energías renovables son recursos energéticos que se encuentran disponibles de forma abundante en la naturaleza. El mayor potencial renovable corresponde a la energía solar, sin embargo, el comportamiento de la radiación en las diferentes horas del día, tiene una naturaleza intermitente y en la noche es nulo. Para superar la intermitencia de la generación solar se han desarrollado sistemas híbridos eólico-solar, integrados con sistemas de almacenamiento, que aseguran un suministro fiable y continúo de energía eléctrica a las diferentes cargas durante un periodo de tiempo requerido. Los sistemas de almacenamiento proporcionan a las instalaciones fotovoltaicas de un elevado nivel de confiabilidad. El diseño y dimensionado de una instalación fotovoltaica depende principalmente de la demanda requerida y del recurso solar existente en una localización dada según Bajpai \& Dash (2012); Iverson, Achuthan, Marzocca, \& Aidun (2013).

Algunos casos de estudio de integración de energía solar y eólica para sistemas de bombeo en áreas remotas, en Argelia, o para la evaluación del potencial de uso de la energía solar y eólica en el sector acuícola, en Tailandia, demuestran resultados favorables. Por ejemplo, en el caso de Tailandia, la generación de energía solar se integra en la producción de camarón. La energía solar fotovoltaica proporciona mayor fiabilidad de suministro en comparación con los sistemas eólicos. Asimismo, la fiabilidad del suministro eléctrico se incrementa significativamente al incluir baterías de almacenamiento, que permiten suministrar energía eléctrica durante la noche, cubriendo incluso casi el $100 \%$ del total de la demanda, en comparación con el $54 \%$, obtenido con un sistema sin baterías (Saidi, Harrouz, Colak, Kayisli, \& Bayindir, 2019); (Nookuea, Campana, \& Yan, 2016).

\subsection{Uso de los recursos energéticos en el sector acuícola}

La acuicultura es una actividad intensiva, que debe obtener la mayor cantidad de producción con el menor uso de recursos y un elevado nivel de tecnificación de procesos y control de parámetros como: temperatura, nivel de oxígeno disuelto, $\mathrm{PH}$, turbidez del agua, etc. La variable de mayor importancia es el nivel de oxígeno (Pringle, Handler, \& Pearce, 2017).

El oxígeno puede ser suministrado por difusión directa o por fotosíntesis. En el primer caso, el oxígeno se libera en el agua mediante procesos de aireación (Figura 2), cambio o recirculación de agua. El segundo caso, consiste en un proceso fotosintético de forma natural, generado por el uso de fitoplancton y algas (Goelzer, Avila, \& de Oliveira, 2017); (Romero, Ponce, \& Marcillo).

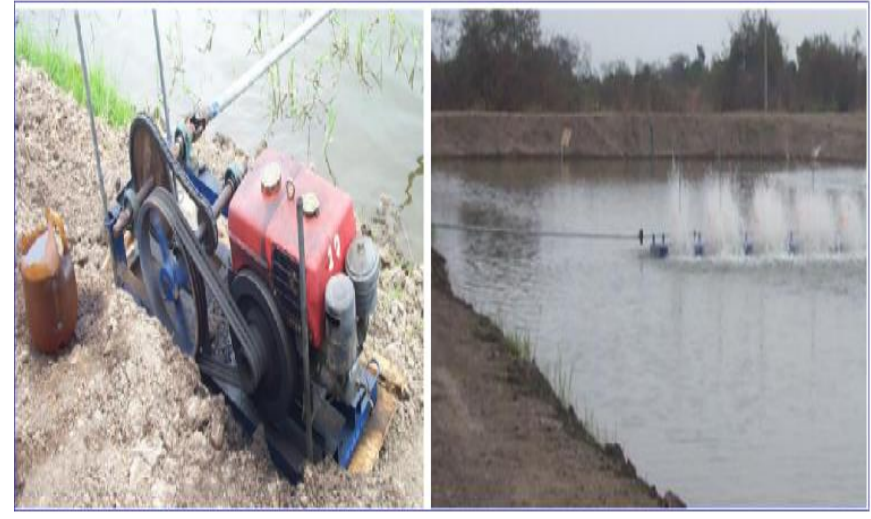

Figura 2. Sistema de aireación con motor diésel

El consumo de recursos energéticos depende en gran medida del tamaño y tipo de cultivo empleado en la camaronera. Por ejemplo, el tipo intensivo se caracteriza por tener piscinas pequeñas, pero con altas densidades de camarones, haciendo imprescindible la instalación de equipos de aireación para mantener los niveles de oxígeno adecuados. En los cultivos de tipo extensivo, caracterizado por tener grandes extensiones, el sistema de bombeo es prioritario para el llenado y recirculación de agua en las piscinas (Figura 3) (Fajardo \& Quevedo, 2018).
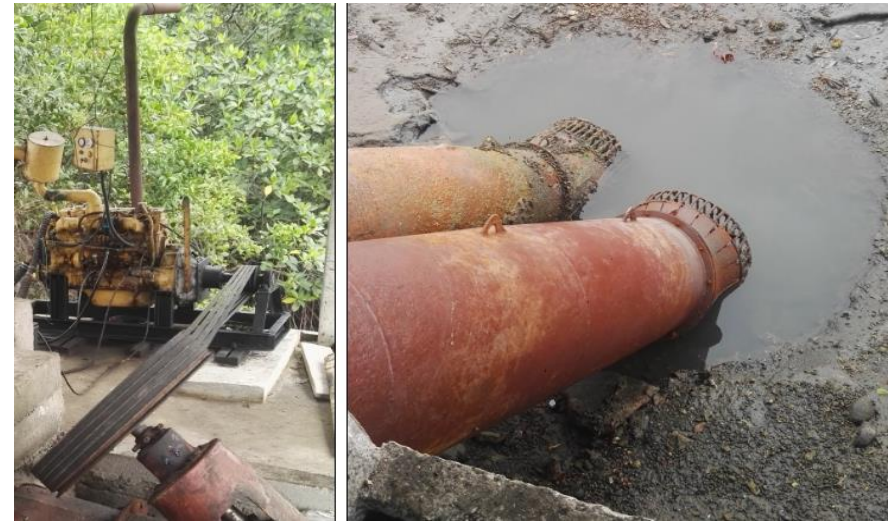

Figura 3. Estación de bombeo diésel de una camaronera

El reemplazo de equipos diésel por eléctricos para los sistemas de aireación presenta varias ventajas, entra las que destacan:

- Se reduce riesgo de contaminación por derrames.

- Reducción de emisión de gases contaminantes.

- No requiere mantenimiento ni cambio de repuestos periódicos.

- Puede ser operado a distancia.

- Menores gastos operativos.

En el caso de los cultivos extensivos, una de las dificultades del manejo y control de parámetros, como el oxígeno, es la gran extensión de las camaroneras. Los trabajadores deben recorrer grandes distancias para encender los equipos de aireación. En los meses de invierno, algunas tareas ya no se realizan por la dificultad de acceso a determinados lugares. Un elevado nivel de tecnificación y el uso de energía eléctrica permiten que estas tareas sean fácilmente automatizadas y monitoreadas en tiempo real, con ayuda de controladores lógicos programables para la gestión a distancia o de forma autónoma. 


\subsection{Tarifas eléctricas para el sector camaronero del Ecuador}

Una ventaja de tener conexión a una red eléctrica pública cercana es el acceso a una tarifa eléctrica diferenciada de menor precio, denominada tarifa de bombeo de agua. Esta tarifa fue publicada en el pliego tarifario del año 2020 por la Agencia de Regulación y Control de Electricidad, ARCONEL. Los rubros de pago que esta tarifa contempla son: comercialización, demanda, y energía horaria diferenciada. La tarifa indica que se deberá aplicar para el uso agrícola y acuícola, siempre y cuando se utilicen elementos eléctricos que formen parte del proceso productivo como oficinas administrativas y guardianía; y siempre y cuando no involucre procesos industriales (Agencia de Regulacion y Control de Electricidad [ARCONEL], 2020).

Para clientes de CNEL El Oro, Los Ríos, Guayas-Los Ríos, Manabí, Milagro, Santa Elena, Santo Domingo y Sucumbíos, la tarifa bombeo de agua posee los valores presentados en la Tabla 1 (ARCONEL, 2020). Las tarifas para clientes en bajo voltaje aplican únicamente cuando la capacidad requerida es menor a $10 \mathrm{~kW}$, en media tensión hasta $1000 \mathrm{~kW}$ y en alta tensión aplica para instalaciones de capacidad superior a 1000 kW (ARCONEL, 2020).

Tabla 1. Tarifa Eléctrica categoría bombeo de agua (ARCONEL, 2020)

\begin{tabular}{lccc}
\hline \multirow{2}{*}{ Rubros } & \multicolumn{3}{c}{ Costo por nivel de voltaje } \\
\cline { 2 - 4 } & Bajo & Medio & Alto \\
\hline Comercialización & 1,414 USD & 1,414 USD & 1,41 USD \\
Demanda & 4,79 USD x & 4,576 USD x & 4,40 USD x \\
Demanda & Demanda & Demanda \\
Energía & 0,07 & 0,061 USD & 0,055 USD \\
08-22 horas & USD.kWh & USD.kWh & USD.kWh \\
Energía & 0,056 & 0,049 USD & 0,049 USD \\
22-08 horas & USD.kWh & USD.kWh & USD.kWh \\
\hline
\end{tabular}

\subsection{Eficiencia energética}

La ISO 50001 es una normativa de gestión energética para sistematizar los procesos en una organización o empresa con el fin de promover criterios de gestión de ahorro y eficiencia energética (Fiedler \& Mircea, 2012).

Los programas de eficiencia energética son una pieza fundamental para lucha contra el cambio climático, con el objetivo de reducir las emisiones de dióxido de carbono a escala global. La norma proporciona una herramienta para lograr la reducción de (Fiedler \& Mircea, 2012).

- Consumo de energía

- Costos de operación

- Emisiones y contaminantes

La gestión energética puede ser aplicada a cualquier tipo de organización que requiera energía para cumplir con sus actividades. Permite además aplicar medidas y actividades que se planifican o ejecutan con el fin de minimizar el consumo energético, considerando incluso patrones de comportamiento de los trabajadores que implique una reducción del consumo de energía y un incremento de la eficiencia energética de la empresa (Fiedler \& Mircea, 2012; Correa, 2016).

El sector acuícola requiere una gran cantidad de energía para realizar sus actividades de producción. La aplicación de mecanismos de eficiencia energética en esta actividad presenta varios beneficios citados a continuación:

- Mejora la eficiencia energética de los procesos.

- Facilita la comunicación y crea transparencia en la gestión de los recursos energéticos.

- Promueve y refuerza los comportamientos y hábitos en cuanto a la gestión de la energía.

- Ayuda a priorizar y evaluar la implementación de nuevas tecnologías energéticamente eficientes.

- Permite integrar otros sistemas de gestión como medio ambiente, salud y seguridad.

- Incrementa el aprovechamiento de energías renovables.

- Asegura la conformidad con una política energética de ahorro y eficiencia energética.

\section{METODOLOGÍA}

Para realizar un estudio de diseño y dimensionado de una instalación solar fotovoltaica en la provincia de El Oro, destinada al suministro eléctrico de una empresa camaronera, es necesario definir el tamaño promedio en hectáreas y tipo de la explotación camaronera. Además, es necesario determinar los valores de radiación solar en los cantones de El Oro.

La información sobre el sector camaronero de la provincia de El Oro se obtiene de la base de datos proporcionada por el Ministerio de Producción, Comercio Exterior, Inversiones y Pesca, MPCEIP. En la provincia de El Oro, existe un total de 996 camaroneras registradas, la mayor cantidad de las cuales se encuentra en el Archipiélago de Jambelí con un total de 498 camaroneras, que representa un $50 \%$ del total del registro existente.

Con la información obtenida del MPCEIP es posible definir las dimensiones promedio de una explotación camaronera tipo extensivo e intensivo, que corresponden a una extensión de 50 Ha y 12 Ha, respectivamente.

Se obtiene información de la radiación solar, temperatura, velocidad del viento de 5 cantones, donde se desarrolla la actividad acuícola en la provincia de El Oro. La evaluación del potencial solar de los sitios estudiados se obtiene a partir de la base meteorológica NREL/NSRDB TMY y de los modelos de radiación del atlas solar del Ecuador, y las estaciones meteorológicas cercanas a los sitios según el anuario del INAMHI, Tabla 2. 
Tabla 2. Sectores Acuícolas de la provincia de El Oro

\begin{tabular}{|c|c|c|}
\hline Lugar & Coordenadas UTM & Altitud \\
\hline Machala & 17 M $615768.55 \mathrm{~m} \mathrm{E}, 9639641.44 \mathrm{~m} \mathrm{~S}$ & $12 \mathrm{~m}$ \\
\hline Jambelí & 17M 595027.67 m E, $9630938.88 \mathrm{~m} \mathrm{~S}$ & $7 \mathrm{~m}$ \\
\hline Arenillas & 17M 601672.82 m E, $9620574.55 \mathrm{~m} \mathrm{~S}$ & $10 \mathrm{~m}$ \\
\hline Huaquillas & 17 M $586473.96 \mathrm{~m} \mathrm{E}, 9618255.51 \mathrm{~m} \mathrm{~S}$ & $11 \mathrm{~m}$ \\
\hline El Guabo & 17 M $620333.10 \mathrm{~m} \mathrm{E}, 9647939.19 \mathrm{~m} \mathrm{~S}$ & $7 \mathrm{~m}$ \\
\hline Sta. Rosa & 17 M $615145.95 \mathrm{~m} \mathrm{E}, 9618327.65 \mathrm{~m} \mathrm{~S}$ & $16 \mathrm{~m}$ \\
\hline
\end{tabular}

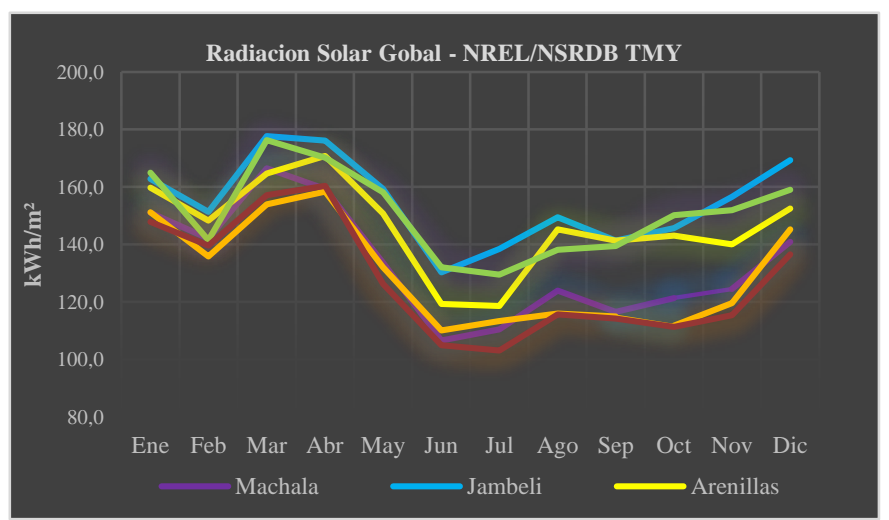

Figura 4. Radiación Solar Global varios sectores Provincia de El Oro

El Cantón Santa Rosa registra niveles de radiación menor en comparación con otros cantones, el mayor potencial solar corresponde al archipiélago de Jambelí. Para efectos del diseño y dimensionado de una instalación fotovoltaica se considerará al cantón de Arenillas, específicamente a Puerto Pitahaya el cual tiene latitud $-3.4323^{\circ}$, puesto que registra un nivel de radiación intermedio (Figura 4).

\subsection{Radiación solar sobre una superficie inclinada}

La potencia fotovoltaica depende de la cantidad de radiación solar incidente sobre los paneles fotovoltaicos. El parámetro más importante para medir la eficiencia de los sistemas fotovoltaicos es el performance ratio, PR, que es la relación entre la energía producida en la salida de alterna del sistema solar y la energía generada por los paneles fotovoltaicos bajo condiciones estándar de ensayo, STC, a un valor de radiación de $1000 \mathrm{~W} / \mathrm{m}^{2}$ y $25^{\circ} \mathrm{C}$. Existen muchos factores que afectan el rendimiento de los sistemas fotovoltaicos como la inclinación, orientación, temperatura, polvo, etc. (Tur, Colak, \& Bayindir, 2018).

Los métodos para el cálculo de irradiaciones sobre superficies inclinadas a partir de datos de irradiación sobre una superficie horizontal son varios, la metodología aplicada se describe a continuación:

Cálculo del ángulo de inclinación: el ángulo óptimo de inclinación se calcula a partir de la latitud del sitio de estudio como se muestra en la Ec. 1 (Lorenzo, 2002).

$$
\beta_{(o p t)}=3.7+0.69 \phi
$$

$\beta_{(o p t)}$ : Ángulo óptimo de inclinación.

$\phi$ : Latitud del emplazamiento.
Orientación o azimut: si el emplazamiento se encuentra en el hemisferio norte se recomienda que el panel se encuentre orientado hacia el sur, y viceversa, de tal forma que se pueda masificar el aprovechamiento radiación solar.

El ángulo de inclinación óptimo teórico para una latitud de $3.4323^{\circ}$ es $6,068^{\circ}$ y $0^{\circ}$ de azimut orientado hacia el norte. Sin embargo, el ángulo práctico de inclinación será $10^{\circ}$, para aprovechar de mejor manera la pendiente por motivos de limpieza de los paneles (Lorenzo, 2002)

La irradiación anual efectiva incidente sobre la superficie del generador $G_{a}(\beta, \alpha)$ se calcula mediante la Ec. (2) (Lorenzo, 2002)

$$
G_{a} \beta_{(o p t)}=G_{a}(0) /\left[1-4.46 \times 10^{-4} \beta_{(o p t)}-1.19 \times 10^{-4} \beta_{(o p t)}^{2}\right]
$$

$$
\begin{aligned}
& G_{a} \beta_{(o p t)} \text { : Irradiación incidente - superficie inclinada } \\
& G_{a}(0) \text { : Irradiación incidente - superficie horizontal. }
\end{aligned}
$$

Considerando una orientación de $0^{\circ}$ y una inclinación de $10^{\circ}$ en Arenillas provincia de El Oro se obtienen los resultados de la irradiación anual efectiva sobre los paneles (Tabla 3). El mes con menor radiación solar es noviembre con $90,78 \mathrm{kWh} / \mathrm{m}^{2}$ mes, equivalente a $3,03 \mathrm{kWh} / \mathrm{m}^{2}$-dia. Por otro lado, el mes con mayor radiación solar es abril con $179,59 \mathrm{kWh} / \mathrm{m}^{2}$-mes, equivalente a $5,98 \mathrm{kWh} / \mathrm{m}^{2}$-dia. La radiación promedio mensual es $136,29 \mathrm{kWh} / \mathrm{m}^{2}$-mes, igual a una radiación diaria de $4,54 \mathrm{kWh} / \mathrm{m}^{2}$-dia.

Tabla 3. Irradiación anual efectiva incidente sobre un panel solar

\begin{tabular}{cccc}
\hline Mes & $\begin{array}{c}\text { NREL } \\
{\left[\mathrm{kWh} / \mathrm{m}^{2} \mathrm{mes}\right]}\end{array}$ & $\begin{array}{c}\text { Atlas Solar } \\
{\left[\mathrm{kWh} / \mathrm{m}^{2} \mathrm{mes}\right]}\end{array}$ & $\begin{array}{c}\text { Irradiacion }(\beta, \alpha) \\
{\left[\mathrm{kWh} / \mathrm{m}^{2} \mathrm{mes}\right]}\end{array}$ \\
\hline Enero & 159,8 & 149,83 & 146,25 \\
Febrero & 148,3 & 135,80 & 132,55 \\
Marzo & 164,7 & 177,90 & 173,65 \\
Abril & 170,8 & 183,99 & 179,59 \\
Mayo & 150,7 & 158,63 & 154,83 \\
Junio & 119,3 & 125,14 & 122,15 \\
Julio & 118,6 & 136,30 & 133,04 \\
Agosto & 145,3 & 131,06 & 127,92 \\
Septiembre & 141,3 & 109,75 & 107,13 \\
Octubre & 143,1 & 99,94 & 97,55 \\
Noviembre & 140,1 & 93,01 & 90,78 \\
Diciembre & 152,5 & 134,10 & 130,89 \\
\hline Anual & $\mathbf{1 7 5 4 , 5 0}$ & $\mathbf{1 6 3 5 , 4 6}$ & $\mathbf{1 5 9 6 , 3 2}$ \\
\hline
\end{tabular}

\subsection{Demanda eléctrica}

La demanda energética requerida para las camaroneras es utilizada para los sistemas de bombeo, los sistemas de aireación y las instalaciones de los campamentos. Generalmente, las fincas camaroneras se encuentran en sitios alejados, por tanto, no disponen de conexión con la red eléctrica pública y satisfacen su demanda de energía mediante el uso de motores diésel para bombeo o aireación y generadores diésel para suministro eléctrico de las instalaciones eléctricas. El empleo de motores de combustión implica un elevado riesgo de contaminación medioambiental, 
que causar graves daños a la evolución del cultivo de los camarones (Fajardo \& Quevedo, 2018; Navia, 2018).

La demanda eléctrica para la estación de bombeo se calcula a partir de la potencia hidráulica necesaria para abastecer las piscinas de cultivo de larvas y crecimiento de camarones y el recambio de agua diario. Para una camaronera de tipo intensivo, el recambio de agua a considerar será del 5\% del volumen de la piscina. En tanto, que para una camaronera de tipo extensiva se considerará un recambio de agua del 15\% del volumen total de la piscina (Calderón \& Sonnenholzner, 2003); (Sánchez \& Torres, 2011).

La potencia necesaria para las estaciones de bombeo se calcula en función el caudal requerido (Ec. 3), que depende directamente del volumen de agua en las piscinas de la camaronera. Una vez se ha calculado el caudal de las bombas, es posible determinar el valor de la potencia hidráulica (Ec. 4) (Fajardo \& Quevedo, 2018)

Donde:

$$
Q=\frac{A \cdot h \cdot \mathrm{R}}{t}
$$

$Q:$ Caudal $\left[\mathrm{m}^{3} / \mathrm{s}\right]$

$A$ : área $\left[\mathrm{m}^{2}\right]$

$h$ : Altura requerida del espejo de agua

$R$ : porcentaje de recambio de agua

$t$ : tiempo [s]

$$
P H=\frac{Q \cdot A D T M B \cdot 60}{4560 \cdot \eta}
$$

Donde:

$P_{H}$ : Potencia hidráulica [HP]

$Q$ : Caudal $\left[\mathrm{m}^{3} / \mathrm{s}\right]$

$A D T M B$ : Altura dinámica de marea baja $[\mathrm{m}]$ $\eta$ : eficiencia

Para un sistema de aireación de piscinas intensivas se considera un valor entre 32 y $40 \mathrm{HP}$ por hectárea. En tanto, que para cultivos extensivos se estiman un valor de $2 \mathrm{HP}$ por hectárea. Se asume que son equipos de aireación con un rendimiento estándar, en término anglosajones se conoce como standart aeration efficiency, SAE, con un valor que sueles oscilar entre $1.5-2.2 \mathrm{~kg} \mathrm{O} / \mathrm{kW}$-h (Calderón \& Sonnenholzner, 2003).

La Tabla 4 muestra los valores de demanda eléctrica requerida para los diferentes procesos de la producción de camarones en función del tipo de producción ya sea esta intensiva o extensiva. El consumo eléctrico del sistema de aireación representa la carga más importante de la instalación en cultivos intensivos, en tanto que, en cultivos extensivos lo son los sistemas de bombeo, ya que en camaroneras extensivas será necesario bombear mucha más agua para llenar las piscinas, mientras que en los sistemas intensivos debido a la densidad de siembra es indispensable mantener los niveles de oxígeno adecuados mediante aireadores.
Tabla 4. Eficiencia y Costos de Inversión en Centrales

\begin{tabular}{lcccc}
\hline \multicolumn{2}{c}{ Sistema } & \multicolumn{2}{c}{ Intensivo } & \multicolumn{2}{c}{ Extensivo } \\
& {$[\mathrm{kW}]$} & {$[\mathrm{kWh}-\mathrm{día}]$} & {$[\mathrm{kW}]$} & {$[\mathrm{kWh}-$ día $]$} \\
\hline Bombeo & 19,40 & 58,20 & 259,00 & 1657,60 \\
Aireación & 286,46 & 2750,02 & 74,60 & 716,16 \\
Pre-cría & 14,92 & 179,04 & 7,46 & 89,52 \\
Campamento & 0,98 & 4,90 & 2,80 & 14,00 \\
\hline Total & $\mathbf{3 2 1 , 7 6}$ & $\mathbf{2 9 9 2 , 1 6}$ & $\mathbf{3 4 3 , 8 6}$ & $\mathbf{2 4 7 7 , 2 8}$ \\
\hline
\end{tabular}

La Figura 5 muestra la curva de demanda eléctrica diaria para los casos de cultivo intensivo y extensivo, en el caso de las camaroneras intensivas la curva de consumo es mucho más plana en comparación con la curva del sistema extensivo, debido a que los sistemas de aireación que representan su carga más alta funcionan de forma prácticamente continúa durante toda la producción, mientras que el sistema de bombeo en las camaroneras extensivas opera únicamente cuando existe marea alta o si se requiere recirculación del agua.

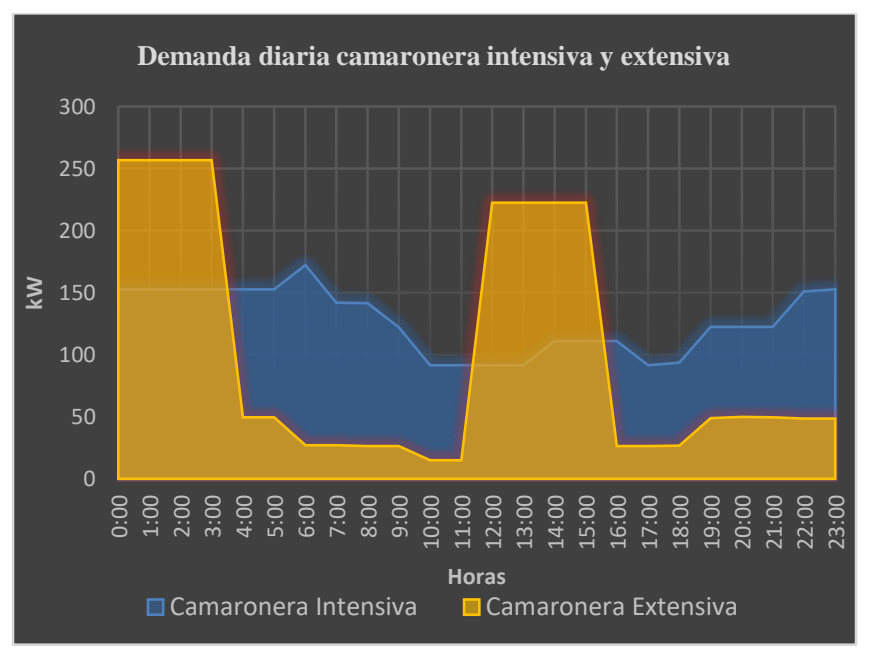

Figura 5. Demanda diaria camaroneras intensivas y extensivas

\subsection{Diseño y dimensionamiento de la instalación fotovoltaica}

El diseño de un sistema solar fotovoltaico aislado se realiza en función la demanda energética requerida, las características técnicas de paneles, baterías e inversor.

El número de paneles a instalar es directamente proporcional a la energía eléctrica requerida durante un día e inversamente proporcional a la potencia pico del panel fotovoltaico, la radiación promedio diaria del lugar, en el peor día del año y la eficiencia de la instalación solar fotovoltaica aislada (Departamento de Energía Solar del Instituto para la Diversificación y Ahorro de la Energía [IDEA] \& CENSOLAR, 2009).

$$
M=\frac{E c}{\mathrm{P}_{m} \cdot \mathrm{hsp} \cdot \eta}
$$

Donde:

M: Numero de módulos FV

$E_{c}$ : Energía consumida diaria, [Wh].

$p_{m}$ : Potencia del módulo, [W].

hsp: Horas sol pico promedio [h].

$\eta$ : eficiencia de la instalación fotovoltaica. 
La radiación promedio diaria se define en función del registro promedio anual, considerando el correspondiente ajuste debido a las perdidas por inclinación y orientación para evitar sobredimensionar o subdimensionar el sistema solar (Tabla 3).

La potencia máxima del módulo se calcula a partir de las condiciones de trabajo, en particular la temperatura ya que de esta depende el desempeño del módulo fotovoltaico, este registro corresponde a la base de datos del Instituto Nacional de Meteorología e Hidrología (INAMHI). El rango de temperatura anual oscila en el lugar seleccionado entre $19,2^{\circ} \mathrm{C}$ y $33,8^{\circ} \mathrm{C}$.

El arreglo del número de paneles solares fotovoltaicos por string y número de strings en paralelo no deberá superar los valores máximos y mínimos del rango de operación de tensión y corriente del inversor.

El diseño propuesto de la instalación solar consiste en un inversor central, que se encargará de suministrar la energía eléctrica generada a la red interna de la instalación de producción de camarones (Tabla 5).

El sistema de acumulación, conformada por baterías estacionarias, se calcula con la Ec. 6 (Fotovoltaica, 2009).

$$
C_{24}=\frac{A \cdot \mathrm{L}_{D}}{P D_{\max } \cdot \eta_{i n v} \cdot \eta_{b a t}}
$$

$C_{24}$ : Capacidad del acumulador [Ah].

A: Autonomía del sistema en días.

$\mathrm{L}_{D}$ : Consumo diario de la carga [Ah].

$P D_{\max }$ : Profundidad de descarga.

$\eta_{i n v}$ : Eficiencia del inversor.

$\eta_{b a t}$ : Eficiencia de las baterías.

La capacidad del sistema de acumulación se calcula considerando una autonomía de 1 día, debido a que el sistema va a ser utilizado durante las horas de la noche como se muestra en la curva de carga en la Figura 4.

Las características de los elementos de la instalación solar fotovoltaica aislada se muestran en la Tabla 5.

Tabla 5. Especificaciones para las instalaciones fotovoltaicas

\begin{tabular}{lcc}
\hline \multicolumn{1}{c}{ Tipo } & Intensiva & Extensiva \\
\hline Inversores & SMA SC560HE & SMA SC500HE \\
Potencia & $560[\mathrm{~kW}]$ & $500[\mathrm{~kW}]$ \\
Rango de voltaje & $480-820[\mathrm{~V}]$ & $480-820[\mathrm{~V}]$ \\
\hline Módulos PV & EGE 72 Cells & EGE 72 Cells \\
Potencia & $350[\mathrm{~W}]$ & $350[\mathrm{~W}]$ \\
Dimensiones & $1956 \times 992 \times 40 \mathrm{~mm}$ & $1956 \times 992 \times 40 \mathrm{~mm}$ \\
Módulos/strings & 14 & 14 \\
Strings & 137 & 114 \\
Número de paneles & 1918 & 1596 \\
Área de los módulos & $3739.86\left[\mathrm{~m}^{2}\right]$ & $3112.00\left[\mathrm{~m}{ }^{2}\right]$ \\
\hline Acumuladores & HOPPECKE OPsZ & HOPPECKE OPsZ \\
& $30 \times 48 \mathrm{~V} 4004$ Ah & $24 \times 48 \mathrm{~V} 4004 \mathrm{Ah}$ \\
Energía Efectiva & $919,24[\mathrm{MWh}-\mathrm{año}]$ & $750,59[\mathrm{MWh}-\mathrm{año}]$ \\
\hline Performance R. & 0,8373 & 0,8216 \\
\hline
\end{tabular}

En la Figura 5 se compara mensualmente la demanda eléctrica, aportación solar y energía auxiliar necesaria para satisfacer el consumo eléctrico de una finca camaronera de tipo intensivo, considerando las características técnicas de la instalación solar aislada propuesta (Tabla 4). En los meses de junio a diciembre se requerirá energía auxiliar. El mes más crítico es noviembre, ya que existe un déficit de generación del 36\%, que deberá ser cubierto por los equipos diésel. Durante los meses de marzo, abril y mayo, se prevé que la generación solar sea superior a la demanda. En abril se estima una generación solar mayor en un $27 \%$ a la demanda, un exceso que se empleará en otros usos.

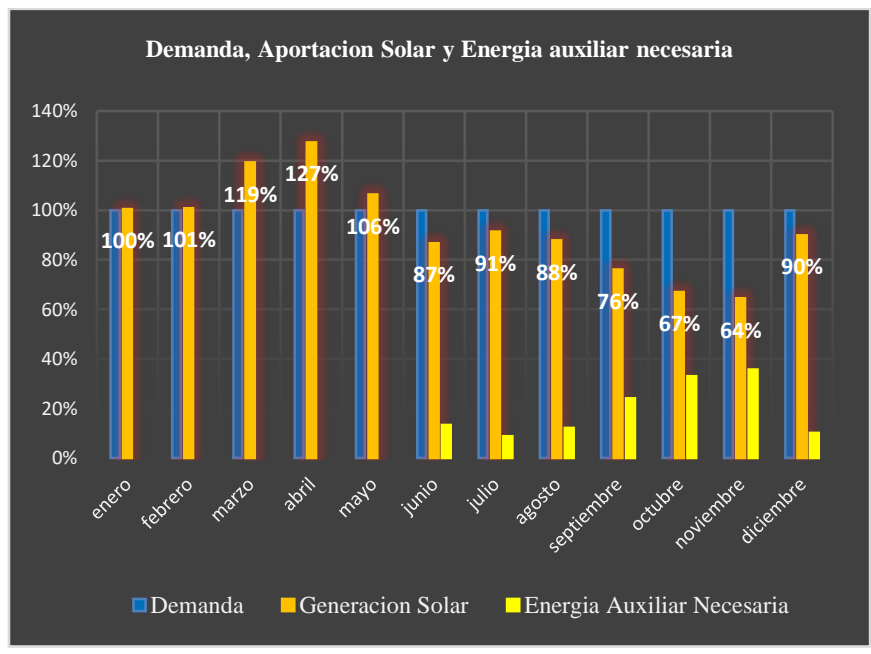

Figura 6. Aportación Solar, demanda y energía auxiliar

Si bien es cierto se ha considerado una demanda mensual constante durante todo el año (Figura 6), en la práctica, la demanda no es constante, ya que el proceso de producción es cíclico se realizan entre 3 y 4 cosechas al año, en este sentido la información del recurso solar es de gran importancia para una correcta planificación del consumo eléctrico y la producción.

\subsection{Análisis Económico}

La Figura 6 muestra los costos de inversión y mantenimiento de las tecnologías solar, red eléctrica y diésel. En el año 0, se presentan los costos de inversión en forma de bloques, mientras que mediante líneas se representa la proyección de los gastos mensuales por operación y mantenimiento de las diferentes tecnologías. El costo de la inversión, así como la operación y mantenimiento de la planta solar se calculan en base a los costos nivelados.

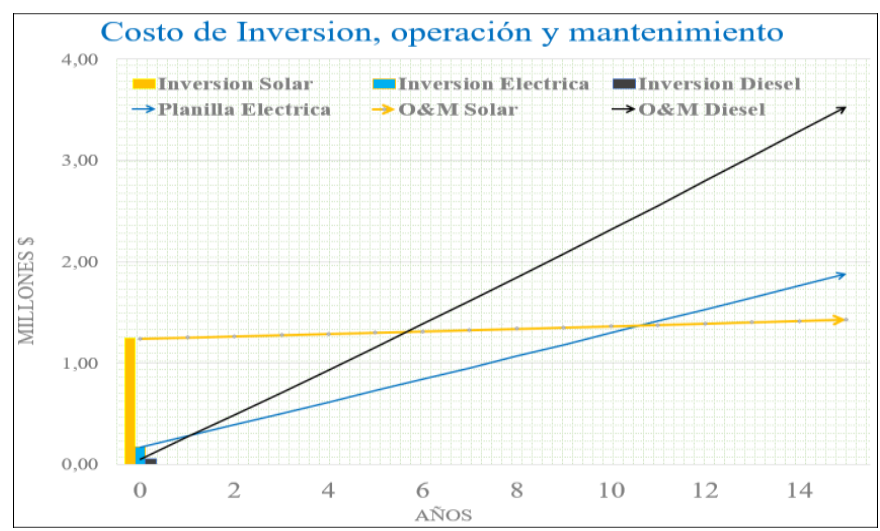

Figura 7. Costos de inversión, operación y mantenimiento 
El periodo de recuperación de la inversión es menor si se compara la tecnología Solar PV vs diésel (Tabla 6). La recuperación de la inversión solar se realiza en un periodo entre 4 y 5 años, mientras que en el caso de la comparación entre Solar PV vs red eléctrica, el periodo de recuperación es más largo, entre 8 y 10 años (Figura 7). Las variables que han sido consideradas son el costo de inversión, mantenimiento, además en el caso del sistema eléctrico dependiente de la red pública se incluye el consto de la planilla eléctrica, para el sistema con motores de combustión interna se considera también el combustible, repuestos, transporte; ya que son rubros considerables en cada tecnología.

Tabla 6. Periodo de recuperación de la inversión

\begin{tabular}{lcc}
\hline Tecnología Actual & $\begin{array}{c}\text { C. Intensiva } \\
\text { Solar PV }\end{array}$ & $\begin{array}{c}\text { C. Extensiva } \\
\text { Solar PV }\end{array}$ \\
\hline Eléctrico & 10 años 299 días & 8 años 142 días \\
Diésel & 5 años 247 días & 4 años 232 días \\
\hline
\end{tabular}

El cálculo del valor actual neto (VAN) resulta de la comparación del flujo costo anual de la tecnología diésel actual versus los costos de la tecnología solar, que implica una inversión inicial elevada, pero con gastos de operación mínimos en comparación con el diésel.

Tabla 7. Tasa de interés, VAN, TIR

\begin{tabular}{|c|c|c|}
\hline Tipo de Camaronera & Intensiva & Extensiva \\
\hline Tasa de interés & $11,58 \%$ & $11,58 \%$ \\
\hline VAN & $\$ 531.613,97$ & $\$ 762.447,08$ \\
\hline TIR & $17,05 \%$ & $20,85 \%$ \\
\hline
\end{tabular}

En la Tabla 7, se muestran los resultados del análisis económico financiero de la instalación solar. El análisis se ha realizado para un periodo de 25 años, en función de la vida técnica para proyectos solares fotovoltaicos (Cisterna et al., 2020). Los resultados más satisfactorios se presentan en el caso de la camaronera extensiva ya que el VAN es mayor, en comparación con el VAN de la camaronera intensiva. Asimismo, en los dos casos evaluados, los proyectos son factibles económicamente puesto que el TIR es mayor a la tasa de interés.

\section{CONCLUSIONES Y RECOMENDACIONES}

Los resultados obtenidos de la integración de sistemas solares fotovoltaicos aislados en la producción camaronera indican que es técnicamente viable el suministro de un importante porcentaje de volumen de energía eléctrica anual para satisfacer la demanda de los equipos de bombeo y aireadores de las fincas camaroneras. La provincia de El Oro, además, presenta excelentes recursos solares que viabilizan la instalación de sistemas solares fotovoltaicos en el sector camaronero del Ecuador.

Los resultados económicos muestran que los proyectos de energía solar fotovoltaica para el sector acuícola intensivo y extensivo son rentables. El VAN evaluado de una instalación fotovoltaica resulta positivo. Asimismo, el periodo de recuperación de la inversión es aproximadamente de 5 años. Por otra parte, los resultados muestran que el sistema tipo extensivo presenta un periodo de recuperación de la inversión más corto, en comparación con el sistema tipo extensivo, debido a que la capacidad solar a instalar y, por tanto, la inversión esperada es menor.

Los sistemas de producción camaronera, poseen actividades multidisciplinarias, y contemplan un gran número de riesgos ya que la producción del crustáceo es altamente sensible a variaciones de parámetros como temperatura, oxigeno, calidad del agua, PH, etc. La tecnificación en estos procesos es crucial, puesto que mejoraría el monitoreo de la producción para la toma de decisiones y a la vez evita el uso de combustibles fósiles, mismos que podrían causar graves daños en la evolución de los animales.

El proceso de producción acuícola requiere gran control de los diferentes parámetros, es primordial evitar que existan contaminantes, puesto que la producción camaronera es muy susceptible, es necesario eliminar los focos potenciales de peligro para reducir el riesgo de daño ecológico y pérdida de la producción. Los equipos eléctricos como transformadores deben ser sumergidos en aceite vegetal dieléctrico biodegradable, utilizado habitualmente en industrias alimenticias, cerveceras, viñedos, etc.

La confiabilidad de los sistemas eléctricos en las instalaciones camaroneras es de gran importancia, puesto que estas fincas acuícolas están ubicadas generalmente a grandes distancias de los centros poblados. Por tanto, los tiempos de respuesta para atender las contingencias suelen ser relativamente largos, sumado al difícil acceso en tiempos invernales complica aún más el tiempo de las interrupciones.

Los proyectos de energías renovables, electrificación y tecnificación están calificados para acceder a certificaciones promovidas por el ministerio del ambiente, que otorgan beneficios a las empresas preocupadas por alcanzar el desarrollo sostenible y la reducción de contaminación ambiental. Estas certificaciones se aplican para las empresas que cumplan con actividades de producción limpia, en las cuales se considera varios puntos como: la innovación y el cambio de tecnología para reducir el riesgo de las personas y el ambiente.

Las plantas solares fotovoltaicas son una alternativa efectiva para la producción de energía en el sector camaronero y garantiza el desacoplamiento económico de este sector productivo de la dependencia del petróleo en el Ecuador.

\section{AGRADECIMIENTOS}

Los autores del artículo quieren agradecer de forma especial a la Universidad Católica de Cuenca, por su apoyo en la realización del presente estudio dentro del programa de Maestría en Energías Renovables. 


\section{REFERENCIAS}

Aguilar, S. E., \& Parrales, G. L. (2015). Estudio de factibilidad de la implementación del Método Raceways en la Camaronera Ipeca [Tesis de Pregrado, Universidad Católica de Santiago de Guayaquil]. Retrieved from http://repositorio.ucsg.edu.ec/handle/3317/4758

Agencia de Regulacion y Control de Electricidad [ARCONEL] (2020). Plieg Tarifario 2020. 35 Retrieved from https://www.cnelep.gob.ec/wpcontent/uploads/2020/01/pliego_tarifario_del_spee_ 2020_resolucion_nro_035_19.pdf

Assad, L. T., \& Bursztyn, M. (2000). Aquicultura no Brasil: bases para um desenvolvimento sustentável. doi:https://doi.org/10.1590/S151635982009001300006

Bajpai, P., \& Dash, V. (2012). Hybrid renewable energy systems for power generation in stand-alone applications: A review. Renewable and Sustainable Energy Reviews, 16(5), 2926-2939. doi:https://doi.org/10.1016/j.rser.2012.02.009

Calderón, J., \& Sonnenholzner, S. (2003). Cultivo de camarón: Experiencia y desafíos en el uso de invernaderos. El mundo acuícola, 9, 4-8.

Cisterna, L., Améstica-Rivas L., \& Piderit, B. (2020). Proyectos Fotovoltaicos en Generación Distribuidai Rentabilidad Privada o Sustentabilidad Ambiental? Revista Politécnica, 45(2), 31-40. doi:https://doi.org/10.33333/rp.vol45n2.03

Correa, A. E. (2016). Propuesta para la implementación de un sistema de gestión integral de la energía en la planta de Colombates SA. Maestría en Administración. Retrieved https://repositorio.unal.edu.co/handle/unal/57585

Fajardo, R. C., \& Quevedo, C. E. (2018). Análisis de factibilidad para la sustitución del uso de energía térmica a energía eléctrica en las camaroneras del cantón Machala. [Tesis de Pregrado, Universidad de Cuenca]

http://dspace.ucuenca.edu.ec/handle/123456789/313 19

Fiedler, T., \& Mircea, P. M. (2012). Energy management systems according to the ISO 50001 standardChallenges and benefits. Paper presented at the 2012 International Conference on Applied and Theoretical Electricity (ICATE) (pp. 1-4). IEEE.

Departamento de Energía Solar del Instituto para la Diversificación y Ahorro de la Energía [IDEA] \& CENSOLAR (2009). Pliego de Condiciones Técnicas de Instalaciones Aisladas de Red. Instituto para la Diversificación y Ahorro de la Energía (IDAE).

Gaudry, K.-H., Godoy-Vaca, L., Espinoza, S., Fernández, G., \& Lobato-Cordero, A. (2019). Normativas de energía en edificaciones ante el cambio climático. ACI Avances en Ciencias e Ingenierías, 11(2). doi:https://doi.org/10.18272/aci.v11i2.1285
Goelzer, R. C., Avila, L., \& de Oliveira, V. M. (2017). Project and Trajectory Control of an Autonomous Aerator for Aquaculture. Paper presented at the Iberian Robotics conference.

Intriago, L. (2016). Análisis del uso de la fuente de energía en la industria acuícola [Tesis de Maestría, Universidad de Guayaquil]. Retrieved from http://repositorio.ug.edu.ec/handle/redug/15722

Iverson, Z., Achuthan, A., Marzocca, P., \& Aidun, D. (2013). Optimal design of hybrid renewable energy systems (HRES) using hydrogen storage technology for data center applications. Renewable energy, 52, 79-87. doi:https://doi.org/10.1016/j.renene.2012.10.038

Lorenzo, E. (2002). La energía que producen los sistemas fotovoltaicos conectados a la red: El mito del $1300 \mathrm{y}$ el "cascabel del gato". Era Solar, 107, 22-28. Retrieved from La energía que producen los sistemas fotovoltaicos conectados a la red: El mito del $1300 \mathrm{y}$ el "cascabel del gato"

McKane, A. (2009). Thinking Globally: How ISO 50001Energy Management can make industrial energy efficiency standard practice.

Navia, V. R. (2018). Análisis comparativo de la producción camaronera en agua dulce y salada en el cantón jama [Tesis de Pregrado, Universidad de Especialidades Espirtu Santo]. https://www.bibliotecasdelecuador.com/Record/oai:1 ocalhost:123456789-2890/Description\#holdings.

Nookuea, W., Campana, P. E., \& Yan, J. (2016). Evaluation of solar PV and wind alternatives for self renewable energy supply: Case study of shrimp cultivation. Energy Procedia, 88, 462-469. doi: 10.1016/j.egypro.2016.06.026

Ochoa, C. A. V. (2016). Programa de bioseguridad para la cría de camarón orgánico Litopenaeus vannamei en cautiverio. Revista AquaTIC(21).

Pesca, M. d. P. C. E. I. y. (2020). Retrieved from https://www.produccion.gob.ec/

Pringle, A. M., Handler, R., \& Pearce, J. M. (2017). Aquavoltaics: Synergies for dual use of water area for solar photovoltaic electricity generation and aquaculture. Renewable and Sustainable Energy Reviews, $\quad 80, \quad 572-584$. doi:https://doi.org/10.1016/j.rser.2017.05.191

Romero, L., Ponce, W., \& Marcillo, M. F. Caracterización y Propuesta Técnica de la Acuacultura en la Zona de El Cajas, Provincia del Azuay. In: Ecuador.

Saidi, A., Harrouz, A., Colak, I., Kayisli, K., \& Bayindir, R. (2019). Performance Enhancement of Hybrid Solar PV-Wind System Based on Fuzzy Power Management Strategy: A Case Study. Paper presented at the 20197th International Conference on Smart Grid (icSmartGrid).

Sánchez, C. R., \& Torres, E. (2011). Comparación de crecimiento de postlarvas de camarón Litopenaeusvannamei con recambio de agua del $15 \%$ 
diario vs recambio de agua necesarios, en los primeros 30 días del cultivo [Tesis de Pregrado, Universidad Nacional Autónoma de Nicaragua]. http://riul.unanleon.edu.ni:8080/jspui/bitstream/123 456789/6509/1/237989.pdf

Haz V. (02 de enero de 2019). Ecuador crecio 1.4\% en el tercer trimestre de 2018. El telegrafo. Retrieved from https://www.eltelegrafo.com.ec/noticias/economia/4/ ecuador-economia-2018

Haz V. (12 de enero de 2019). Gobierno y camaroneros alcanzaron acuerdos sobre el precio del combustible. $\mathrm{El}$ telégrafo. https://www.eltelegrafo.com.ec/noticias/economia/4/ gobierno-camaroneros-acuerdo-diesel

Tur, M. R., Colak, İ., \& Bayindir, R. (2018). Effect of Faults in Solar Panels on Production Rate and Efficiency. Paper presented at the 2018 International Conference on Smart Grid (icSmartGrid).

Vieira$^{1}$, D. M., de Oliveira, M. A., Crispim, M. C., \& Cunha, B. (2016). Por uma aquicultura familiar sustentável: bases jurídicas e da política do setor no Brasil. doi:http://dx.doi.org/10.21707/gs.v10.n04a41

\section{BIOGRAFÍAS}

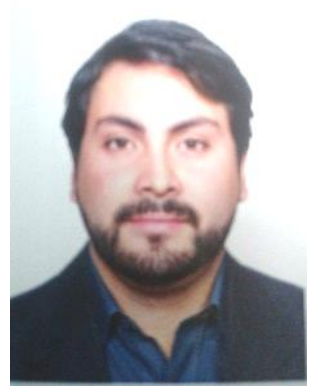

Juan Pablo Pesantez. - Nació en Toronto en 1989. Recibió su título de Ingeniero Eléctrico de la Universidad Politécnica Salesiana en 2016; Actualmente se desempeña como Ingeniero Eléctrico en libre ejercicio, y se encuentra cursando una maestría en Energías Renovables en la Universidad Católica de Cuenca. Sus campos de investigación están relacionados con el Desarrollo de Energías renovables aplicados al sector agrícola y acuícola del Ecuador.

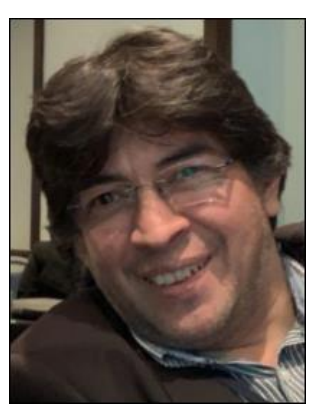

Alberto Ríos Villacorta. - Nació en Lima en el año 1969. Ingeniero Eléctrico, especializado en Sistemas y Redes Eléctricas, Facultad de Energía, Instituto Politécnico de Bielorrusia, Unión de Repúblicas Socialistas Soviéticas, URSS, 1993. Master en Energías Renovables, Universidad Europea de Madrid-Iberdrola, España, 2004. Doctor en Ingeniería Eléctrica, Departamento de Ingeniería Eléctrica, Universidad Carlos III de Madrid, España, 2007. Desde el año 2017 es Profesor Titular Principal de la Universidad Técnica de Ambato. Director del Grupo de Investigación REWA-RD, Renewable Energy \& Web Architecture - Research and Development, Universidad Técnica de Ambato, Ecuador.

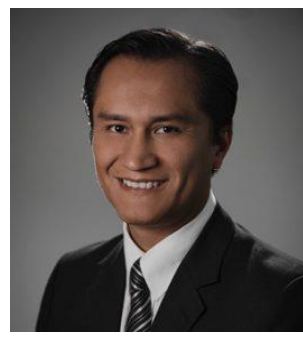

Javier González Redrován. - Nació en Azogues en 1980. Ingeniero electrónico por la Universidad Politécnica Salesiana en 2006. Magíster en Energías Renovables por la Universidad de las Fuerzas Armadas - ESPE en 2015. Actualmente, se desempeña como Docente de Posgrado y Coordinador Académico de la Maestría en Energías Renovables en la Universidad Católica de Cuenca, y como Investigador en proyectos relacionados con energías renovables y generación distribuida. 